\title{
Rare-earth spin-glasses with uniaxial anisotropy
}

\author{
K. Baberschke, ${ }^{*}$ P. Pureur, and A. Fert \\ Laboratoire de Physique des Solides associé au Centre Nationale de la Recherche Scientifique, \\ Université de Paris-Sud, 91405 Orsay Cedex, France
}

R. Wendler

Institut für Atom- und Festkörperphysik, Freie Universität Berlin, Arnimallee 14, D-1000 Berlin 33, West Germany

S. Senoussi

Laboratoire de Physique des Solides associé au Centre Nationale de la Recherche Scientifique, Université de Paris-Sud, 91405 Orsay Cedex, France

(Received 19 December 1983)

\begin{abstract}
The properties of spin-glasses with uniaxial anisotropy are investigated by magnetization measurements on single crystals of $\mathrm{Y}$ and $\mathrm{Sc}$ doped with $\mathrm{Er}, \mathrm{Dy}, \mathrm{Tb}$, or Gd impurities. In alloys with strong anisotropy, spin-glass properties (susceptibility, cusp, irreversibility) appear only for fields along the $c$ axis of the crystal (Ising-like systems) or only for fields in the basal plane ( $X$ - $Y$-like systems). The freezing temperature of the Ising-like systems is higher than that of the Heisenberg systems (alloys with Gd) by about a factor of 3. These results are in agreement with recent theoretical predictions. In alloys with small anisotropy, the irreversibility effects appear at the same temperature in longitudinal and transverse fields and we cannot identify the two-stage freezing predicted by the theory.
\end{abstract}

\section{INTRODUCTION}

The properties of metallic spin-glasses (SG) with uniaxial single-ion anisotropy are beginning to be extensively studied. $^{1-6}$ The starting point of most of the theoretical approaches $^{1,2}$ is an extension of the SherringtonKirkpatrick (SK) Hamiltonian: ${ }^{7}$

$$
\mathscr{H}=-\sum_{i j} J_{i j} \overrightarrow{\mathrm{S}}_{i} \cdot \overrightarrow{\mathrm{S}}_{j}-D \sum_{i} S_{i z}^{2}-g \mu_{B} \overrightarrow{\mathrm{H}} \cdot \sum_{i} \overrightarrow{\mathrm{S}}_{i}
$$

The first term describes the interaction of classical vectors coupled via a set of infinite-range exchanges $\left\{J_{i j}\right\}$ whose probability distribution is

$$
P\left(J_{i j}\right)=\left(\frac{N}{2 \pi j^{2}}\right)^{1 / 2} \exp \left[-N\left(J_{i j} / N\right)^{2} / 2 j^{2}\right] .
$$

The second term of Eq. (1) accounts for a uniaxial crystal field and the third term for the interaction with an applied field. We show in Fig. 1 the phase diagram predicted by Roberts and Bray ${ }^{2}$ and Cragg and Sherrington. ${ }^{1}$

For $D>0$ and $D / j \gg 1$ the Hamiltonian $\mathscr{H}$, Eq. (1), is equivalent to an Ising-type SK Hamiltonian. This is consistent with the top of the phase diagram, Fig. 1, showing a freezing of the longitudinal spin components at $T / j=3$.

For $D<0$ and $|D| / j \gg 1$, the Hamiltonian $\mathscr{H}$ is equivalent to an $X-Y$-type SK Hamiltonian. This is again consistent with the lowest part of the phase diagram, Fig. 1, showing a freezing of the transverse components at $T / j=1.5$.

For $D=0$, the Hamiltonian is a Heisenberg-type SK Hamiltonian, which is consistent with a freezing of both the longitudinal and transverse components at $T / j=1$.

The difference between the values of $T_{g} / j$ in the Ising,
$X-Y$, and Heisenberg limits $(3,1.5$, and 1 , respectively) is a remarkable feature of the mean-field model of spinglasses. ${ }^{7,8}$ Such a difference has never been confirmed by experiments because it is generally very difficult to know the relative values of $j$ in different systems. In this

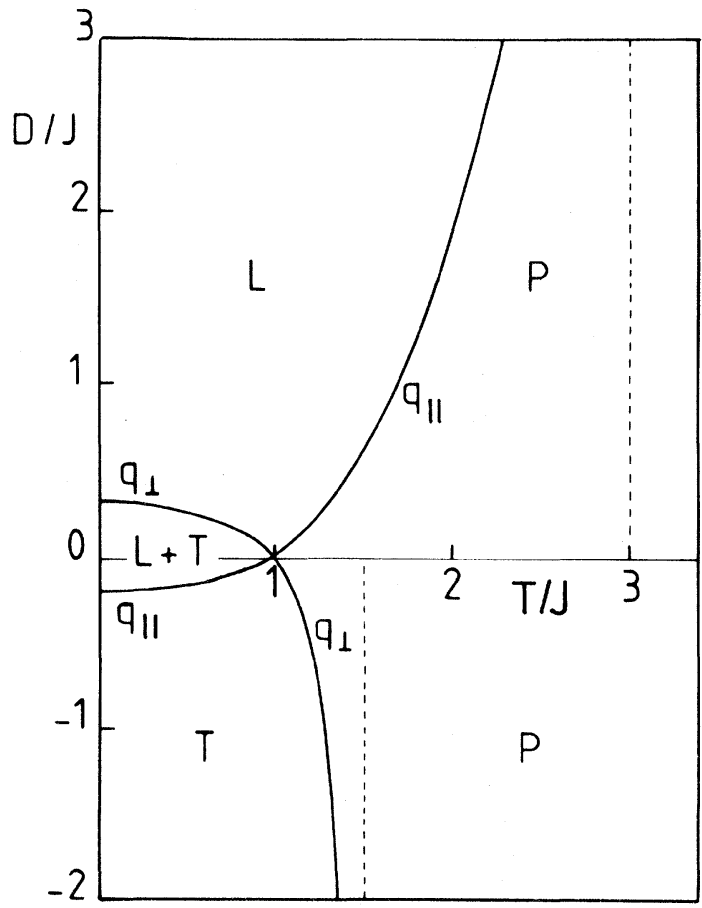

FIG. 1. Schematic phase diagram for a spin-glass with infinite-range interactions and uniaxial anisotropy. For notation see Eqs. (1) and (2) and Refs. 1 and 2. 
respect, the $Y \mathscr{R}$ and $S c \mathscr{R}$ (where $\mathscr{R}$ represents a rareearth element) alloys present two advantages: First, there exist Ising-, $X-Y_{-}$, and Heisenberg-like ions and second, the ratio between the values of $j$ for two different rare earths (RE) in the same host is known. One of the motivations of this work was to study the spin-glass properties in the Ising, $X-Y$, and Heisenberg limits and to probe the theoretical predictions for the relative values of $T_{g} / j$.

For intermediate values of $D / j$ the models of Refs. 1 and 2 describe the progressive change from an Ising or $X-Y$ spin-glass to a Heisenberg spin-glass. At sufficiently large values of $|D| / j$ they predict the freezing of only one spin component (longitudinal for $D>0$, transverse for $D<0$ ). At sufficiently small values of $|D| / j$ they predict the successive freezing of the two components at different temperatures; see the phases $\mathrm{L}$ and LT and T and LT in Fig. 1. How the crossing of the lower phase boundary should show up in magnetization experiments cannot be predicted. One could imagine that irreversibility effects will appear at different temperatures in transverse and longitudinal fields. Alternatively, one could also imagine that the irreversibility effects will appear for both directions at the upper phase boundary. To clarify this aspect of the problem was also a motivation of our work.

We report in this paper on magnetization measurements on single crystals of $\mathrm{Y}$ and Sc containing $\mathrm{Er}, \mathrm{Dy}, \mathrm{Tb}$, and Gd impurities. The crystal-field parameters are well known., ${ }^{9,10} \mathrm{YEr}$ and $\mathrm{ScEr}$ are expected to be Ising-like systems $(D>0)$, while the alloys with $\mathrm{Dy}$ and Tb are expected to be $X$-Y-like $(D<0)$. The ratios $D / j$ can be changed by varying the RE concentration, which allows us to go from the Ising or $X$ - $Y$ limit at low concentrations to intermediate situations at high concentrations. For YGd and $S c G d, D$ is very small $\left(\sim 4 \times 10^{-3} \mathrm{~K}\right)$. Their magnetic properties appear to be not exactly, but almost, isotropic. We shall present these properties and discuss the possible origin of their small anisotropy in a separate publication. In this paper we shall only report on their cusp temperature, which we shall take as characteristic of Heisenberg systems.
This work is similar to the experiments on $\mathrm{Mn}$ in $\mathrm{Zn}$, $\mathrm{Cd}$, and $\mathrm{Mg}$ by Albrecht et al. ${ }^{3}$ An important difference is that we can compare the behavior of different ions in one host while, for example, a quantitative comparison of $\mathrm{ZnMn}$ and $C d \mathrm{Mn}$ is difficult.

The paper is organized in the following way. In Sec. II we adapt the vector spin Hamiltonian, Eq. (1), to our systems containing RE ions with quantum moments $\overrightarrow{\mathbf{J}}$. We present our experimental results in Sec. III and a discussion of these results in Sec. IV. It will be shown that while some of the properties predicted by the theory are found in our experiment, other results are in conflict with the theory. Finally, we would like to stress that the appearance of cooperative magnetic properties in only one or two directions of the lattice structure is not surprising; it follows directly from the physics of single ions. A more important question is to see if the different behaviors observed for different directions are well described by the mean-field spin-glass model.

\section{CRYSTAL-FIELD AND EXCHANGE INTERACTIONS FOR THE RE'S IN Y AND SC}

The crystal-field (CF) Hamiltonian for RE ions in hexagonal symmetry is given by ${ }^{11}$

$\mathscr{H}_{\mathrm{CF}}=B_{2}^{0} O_{2}^{0}(\overrightarrow{\mathrm{J}})+B_{4}^{0} O_{4}^{0}(\overrightarrow{\mathrm{J}})+B_{6}^{0} O_{6}^{0}(\overrightarrow{\mathrm{J}})+B_{6}^{6} O_{6}^{6}(\overrightarrow{\mathrm{J}})$

with $O_{2}^{0} \equiv 3 J_{z}^{2}-J(J+1)$.

We give in Table $I$ the values of the crystal-field coefficients $B_{2}^{0}, B_{4}^{0}, B_{6}^{0}, B_{6}^{6}$ extracted from experiments on dilute $\mathrm{Y}$ - and Sc-based alloys. ${ }^{9,10} B_{2}^{0} O_{2}^{0}$ is the largest term in Eq. (3) and determines the main features of the magnetic behavior, i.e., easy magnetization along the $c$ axis for $B_{20}<0$ or easy magnetization in the basal plane for $B_{20}>0$. The other terms also influence the magnetic properties. For example, the term $B_{6}^{6} O_{6}^{6}$ can mix states $\left|J_{z}=M\right\rangle$ and $\left|J_{z}=M \pm 6\right\rangle$ and thereby changes somewhat the wave function of the ground state (see Table I). However, in general, the influence of the fourth- and sixth-order terms is not very significant. Since we want to compare our experimental results to the predictions of

TABLE I. Crystal-field parameters, overall crystal-field splitting, and wave function of the ground state for several rare-earth ions in Sc or Y (from Ref. 9). $\xi_{J}$ is the de Gennes factor $\left[\xi_{J}=\frac{1}{3} J(J+1)\left(g_{J}-1\right)^{2}\right]$. In $Y$ Tb and $S c$ Tb there are several states in a smallenergy range ( $7 \mathrm{~K}$ in $Y \mathrm{~Tb}, 8 \mathrm{~K}$ in $S c \mathrm{~Tb}$ ) above the lowest level. We give the wave function of all these states.

\begin{tabular}{|c|c|c|c|c|c|c|c|}
\hline $\begin{array}{l}\text { Impurities } \\
\text { (de Gennes factor) }\end{array}$ & Host & $\begin{array}{l}B_{2}^{0} \\
(\mathbf{K})\end{array}$ & $\begin{array}{c}B_{4}^{0} \\
\left(10^{-3} \mathbf{K}\right)\end{array}$ & $\begin{array}{c}B_{6}^{0} \\
\left(10^{-5} \mathrm{~K}\right)\end{array}$ & $\begin{array}{c}B_{6}^{6} \\
\left(10^{-4} \mathrm{~K}\right)\end{array}$ & $\Delta / K$ & Ground state \\
\hline Er & $\mathrm{Y}$ & -0.29 & 0.6 & 2.4 & 2.8 & 123 & $0.97\left|+\frac{13}{2}\right\rangle-0.23\left| \pm \frac{1}{2}\right\rangle+0.12\left| \pm \frac{11}{2}\right\rangle$ \\
\hline$\left(\xi_{J}=2.55\right)$ & $\mathrm{Sc}$ & -0.07 & 0.36 & 3.6 & -2.6 & 156 & $0.83\left| \pm \frac{13}{2}\right\rangle-0.48\left| \pm \frac{1}{2}\right\rangle+0.27\left| \pm \frac{11}{2}\right\rangle$ \\
\hline Dy & Y & 0.33 & -2.8 & 3.8 & -3.6 & 170 & $0.22\left| \pm \frac{13}{2}\right\rangle-0.95\left| \pm \frac{1}{2}\right\rangle+0.20\left|\mp \frac{11}{2}\right\rangle$ \\
\hline$\left(\xi_{J}=7.08\right)$ & Sc & 0.19 & -0.86 & 2.0 & -2.1 & 90 & $0.29\left| \pm \frac{13}{2}\right\rangle-0.92\left| \pm \frac{1}{2}\right\rangle+0.29\left|\mp \frac{11}{2}\right\rangle$ \\
\hline $\mathrm{Tb}$ & $\mathrm{Y}$ & 0.89 & 0.55 & -1.32 & 1.0 & 90 & $\begin{array}{l}0.041| \pm 5\rangle+0.999|\mp 1\rangle \\
0.015| \pm 6\rangle+1.000|0\rangle+0.015|6,-6\rangle \\
0.115| \pm 4\rangle+0.993|6, \mp 2\rangle\end{array}$ \\
\hline$\left(\xi_{J}=10.5\right)$ & Sc & 0.31 & -8.3 & 7.9 & -7.6 & 53 & $\begin{array}{l}0.61| \pm 4\rangle+0.79|\mp 2\rangle \\
0.7|3\rangle+0.7|-3\rangle\end{array}$ \\
\hline
\end{tabular}


TABLE II. $T_{g}, T_{g} / j$, and $D / j$ for our single crystal and polycrystal (p) alloys. $T_{g}$ is the temperature of the splitting between ZFC and FC susceptibility curves in very small fields. $T_{g} / j$ and $D / j$ are calculated as described in Sec. II.

\begin{tabular}{ccccc}
\hline \hline Alloy & $\begin{array}{c}\text { Concentration } \\
\text { (at. \%) }\end{array}$ & $\begin{array}{c}T_{g} \\
(\mathrm{~K})\end{array}$ & $T_{g} / j$ & $D / j$ \\
\hline$Y \mathrm{Er}$ & 2 & 1.93 & 2.71 & 28.4 \\
& 4 & 3.9 & 2.75 & 19.2 \\
& 5 & 5.6 & 3.16 & 11.3 \\
$S c \mathrm{Er}$ & 10 & 3.4 & 3.07 & 4.07 \\
& $15 \mathrm{p}$ & 4.1 & 2.49 & 2.71 \\
& $20 \mathrm{p}$ & 5.4 & 2.46 & 2.03 \\
$Y \mathrm{Dy}$ & 3 & 9.2 & 3.11 & -7.12 \\
$S c \mathrm{Dy}$ & 5 & 4.7 & 3.12 & -7.95 \\
& $9.5 \mathrm{p}$ & 8 & 2.76 & -4.19 \\
& $14.6 \mathrm{p}$ & 11.4 & 2.57 & -2.72 \\
& $19 \mathrm{p}$ & 16.4 & 2.83 & -2.09 \\
$Y \mathrm{~Tb}$ & 1 & 3.2 & 2.2 & -25.6 \\
& $2 \mathrm{p}$ & 8.5 & & \\
$S c \mathrm{~Tb}$ & 4.5 & 6.25 & 3.05 & -6.41 \\
$Y \mathrm{Gd}$ & 1 & 2.1 & 1 & $\sim 0$ \\
$S c \mathrm{Gd}$ & 5 & 3.5 & 1.02 & $\sim 0$ \\
& 15 & 10 & 1.08 & $\sim 0$ \\
\hline \hline
\end{tabular}

models based on Eq. (1), which contains only quadratic $\mathrm{CF}$ terms, we neglect the fourth- and sixth-order terms.

Our total Hamiltonian, including the crystal-field, the exchange, and Zeeman interactions is written as

$$
\mathscr{H}=-\sum_{i j} \Gamma_{i j}\left(g_{J}-1\right)^{2} \overrightarrow{\mathrm{J}}_{i} \cdot \overrightarrow{\mathrm{J}}_{j}+\sum_{i} B_{2}^{0} J_{i z}^{2}-g_{J} \mu_{B} \overrightarrow{\mathrm{H}} \cdot \sum_{i} \overrightarrow{\mathrm{J}}_{i}
$$

We have included the de Gennes coefficient $\left(g_{J}-1\right)^{2}$ in the expression for the exchange term to explicitly show that the exchange coefficient for different RE ions in the same host scales as $\left(g_{J}-1\right)^{2}$. Equation (4) can be rewritten in the form of a classical spin Hamiltonian by replacing the quantum spin operators $\overrightarrow{\mathrm{J}}$ with classical vectors of the same length:

$$
\overrightarrow{\mathbf{J}} \rightarrow \frac{\sqrt{J(J+1)}}{\sqrt{3}} \overrightarrow{\mathbf{S}},
$$

where $\overrightarrow{\mathrm{S}}$ is a vector of length $\sqrt{3}$. A vector spin Hamiltonian of the form of Eq. (1) is thus obtained with

$$
\begin{aligned}
& J_{i j}=\frac{J(J+1)}{3}\left(g_{J}-1\right)^{2} \Gamma_{i j}, \\
& D=-\frac{J(J+1)}{3} B_{2}^{0} .
\end{aligned}
$$

Adopting the conventional assumption of a distribution width of the $\Gamma_{i j}$ exchange coefficients proportional to the concentration $c$, the parameter $j$ is written as

$$
j=c \frac{J(J+1)}{3}\left(g_{J}-1\right)^{2} \Gamma=c \xi_{J} \Gamma,
$$

where the coefficient $\xi_{J}=\frac{1}{3} J(J+1)\left(g_{J}-1\right)^{2}$ depends only on the RE element, while the coefficient $\Gamma$ depends

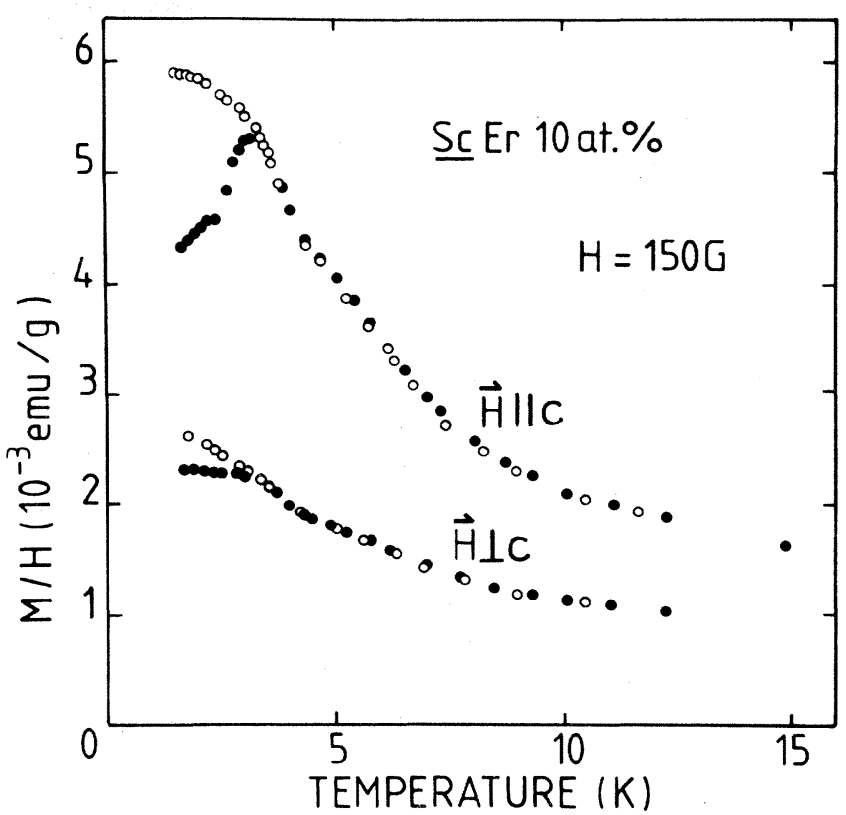

FIG. 2. ZFC (๑) and FC (O) susceptibilities of ScEr 10 at. \% in longitudinal and transverse fields $(150 \mathrm{G})$. The $\mathrm{ZFC}$ and $\mathrm{FC}$ curves split at the temperature of the cusp of $\chi_{\|}^{\mathrm{ZFC}}$.

only on the host (Sc or Y). Finally, the ratios $T / j$ and $D / j$ involved in the phase diagram of Fig. 1 must be replaced by the following ratios:

$$
\begin{aligned}
& T / j \rightarrow T /\left(c \xi_{J} \Gamma\right), \\
& D / j \rightarrow-B_{2}^{0} /\left[c\left(g_{J}-1\right)^{2} \Gamma\right] .
\end{aligned}
$$

$\Gamma$ is a common coefficient for all the RE's in a given host but is unknown. We shall adopt the value of $\Gamma$ giving $T_{g} / j=1$ for the quasi-Heisenberg alloys containing $S$ state ions, i.e., $S c G d$ and $Y G d$. For $S c G d, T_{g}$ is proportional to $c$, so that we can choose $\Gamma_{\mathrm{Sc}}$ so that $T_{g} / j=1$ for all the concentrations. For $Y G d, T_{g}$ is not exactly proportional to $c$ and we have chosen $\Gamma_{\mathrm{Y}}$ so that $T_{g} / j=1$ for YGd 1 at. \%. The values $\Gamma_{Y}$ and $\Gamma_{S c}$ are in the ratio $\Gamma_{\mathrm{Y}} / \Gamma_{\mathrm{Sc}}=3.25$. We show in Table II the values of $D / j$ calculated in the above mentioned way for all our alloys. $D / j$ is positive for the alloys with $\mathrm{Er}$ and negative for the alloys with $\mathrm{Dy}$ and $\mathrm{Tb}$. We see that $|D / j|$ is systematically smaller in the Sc-based alloys than in the Y-based alloys (larger values of $|D / j|$ in Sc-based alloys would imply smaller concentrations, lowering $T_{g}$ and therefore measurements at lower temperatures).

\section{EXPERIMENTAL RESULTS}

We present magnetization measurements on a series of Sc- and Y-based single crystals containing $\mathrm{Er}, \mathrm{Dy}, \mathrm{Tb}$, or Gd impurities. Most of the single crystals have been made by strain annealing. We measured their magnetization by using a Foner-type magnetometer.

\section{A. ScEr and $Y \mathrm{Er}$ (Ising-like alloys)}

We show in Figs. 2 and 3 the field cooling (FC) and zero field cooling (ZFC) susceptibilities of ScEr 10 at. \% 


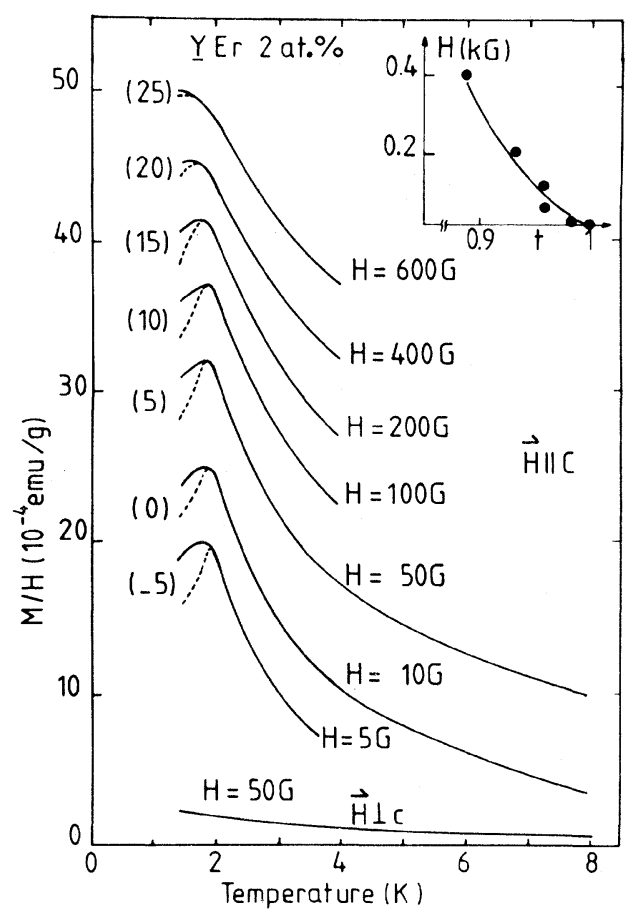

FIG. 3. ZFC (dashed line) and FC (solid line) susceptibilities of $Y \mathrm{Er} 2$ at. $\%$ in longitudinal and transverse fields. The longitudinal susceptibility is shown for several values of the field. The curve for $10 \mathrm{G}$ corresponds to the scale of the vertical axis; the curves for other fields are shown with an offset of $5,10, \ldots$ Inset: Field $H$ vs $T=\Theta / T_{g}$, where $\Theta$ is defined as the temperature of the splitting between the FC and ZFC susceptibility. The solid line corresponds to $H^{2 / 3} \sim 1-t . T_{g}=\Theta(H \rightarrow 0)$.

and $Y \operatorname{Er} 2$ at. \%. For $H$ parallel to the $c$ axis, both alloys exhibit a sharp cusp of the ZFC susceptibility at a temperature $\Theta$ and a splitting of the ZFC and FC curves below $\Theta$. For $H$ in the basal plane, the behaviors of $Y \mathrm{Er}$ and $S c \mathrm{Er}$ are different. The transverse susceptibility of $Y$ Er 2 at. \% does not show any anomaly at the temperature $T_{g}$ where a cusp is observed in the longitudinal susceptibility. Moreover, the FC and ZFC transverse susceptibility coincide down to the lowest temperature in our experiments. In contrast, the transverse $\mathrm{ZFC}$ susceptibility of $S c \mathrm{Er} 10$ at. \% exhibits a bifurcation at the temperature $(\Theta)$ of the cusp of the longitudinal susceptibility and the ZFC curve departs from the FC curve below this temperature.

We have found for all our $Y \mathrm{Er}$ alloys the same behavior as that of $Y \operatorname{Er} 2$ at. \%. Since $D / j$ is large for all these $Y \mathrm{Er}$ alloys (see Table II), their experimental behavior is in agreement with the top of Fig. 1, i.e., with a freezing of only the longitudinal spin components. $D / j$ is definitely smaller for $S c \operatorname{Er} 10$ at. \% (see Table II), so that the existence of freezing in both longitudinal and transverse directions is not surprising. However, our results suggest that the freezing temperature is the same for both directions.

We show the field dependence of $\Theta$ for $Y \operatorname{Er} 2$ at. \% in the inset of Fig. 3. The experimental points are approximately on an Almeida-Thouless-like line corresponding

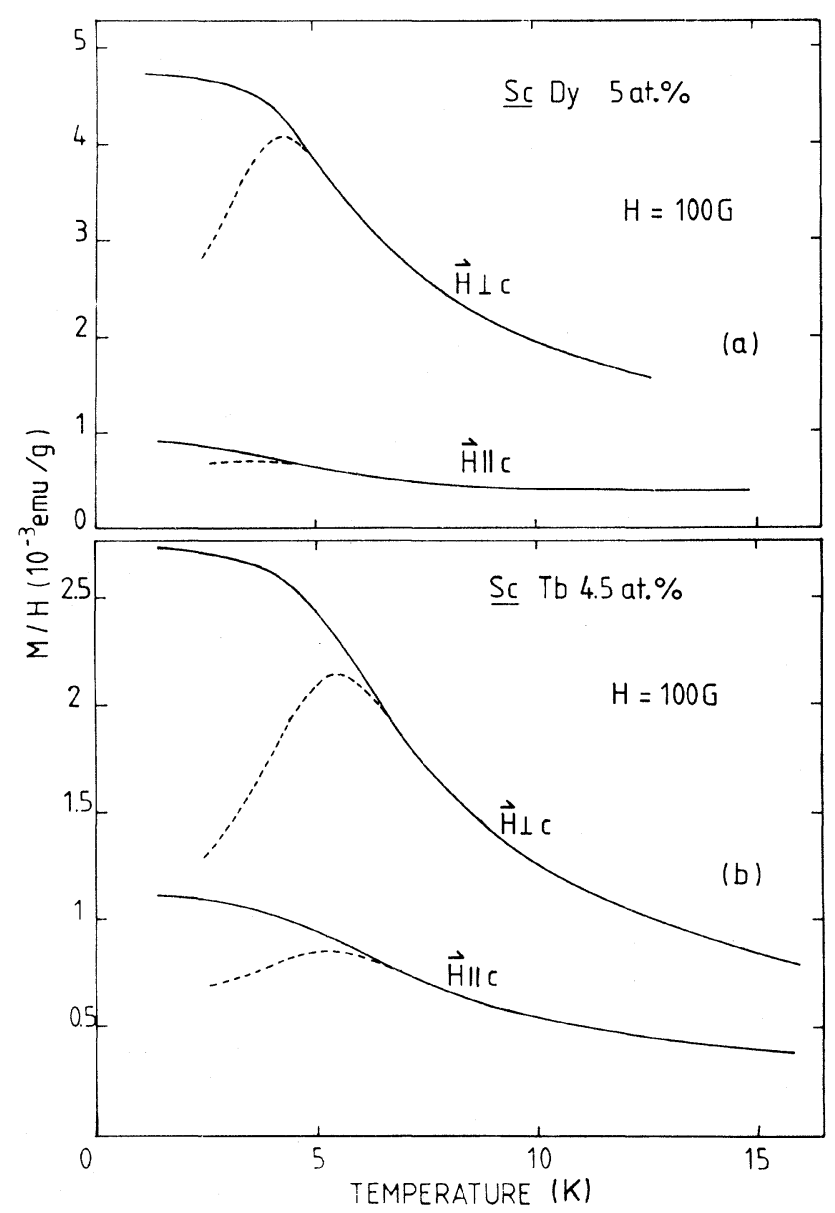

FIG. 4. ZFC (dashed line) and FC (solid line) susceptibilities of (a) $S c D y 5$ at. $\%$ and (b) $S c$ Tb 4.5 at. $\%$ in transverse and longitudinal fields. In both alloys and for both field directions the temperature of the maximum is lower than the temperature where the ZFC curve departs from the FC one.

to $H^{2 / 3} \sim 1-\Theta / T_{g} \cdot{ }^{12,13}$ However, a fit with the theoretical expression of the Almeida-Thouless (AT) line is only obtained with $g_{\text {eff }}=3.3$ for the doublet ground state instead of $g_{\text {eff }}=14.4$ for the ground state of Table II. ${ }^{14}$ This discrepancy of a factor of 4 is surprising in view of the good agreement for $Y \operatorname{Er~} 4$ at. \% ${ }^{6}$ However, one should recall that the theory assumes a Gaussian distribution $P\left(J_{i j}\right)$ and deviations from such a distribution change the scaling. Significant deviations from a Gaussian distribution are likely to exist in $Y \operatorname{Er} 4$ at. \% because, for 4 at. \% impurities in an hcp lattice, the probabilities of having one nearest neighbor (out of 12) or one of the many (32) next-nearest neighbor lattice sites occupied by an impurity are almost equal.

\section{B. $S c D y, S c T b, Y D y$, and $Y T b(X-Y$-like alloys $)$}

We show the FC and ZFC susceptibilities of $Y$ Dy 3 at. \%, $Y \mathrm{~Tb} 1$ at. \%, $S c \mathrm{Dy} 5$ at. \%, and $S c \mathrm{~Tb} 4.5$ at. \% alloys in Figs. 4-6. As for the alloys with Er impurities, there is a different behavior for the Y-based alloys, for which $|D| / j$ is large, and the Sc-based alloys, for which 


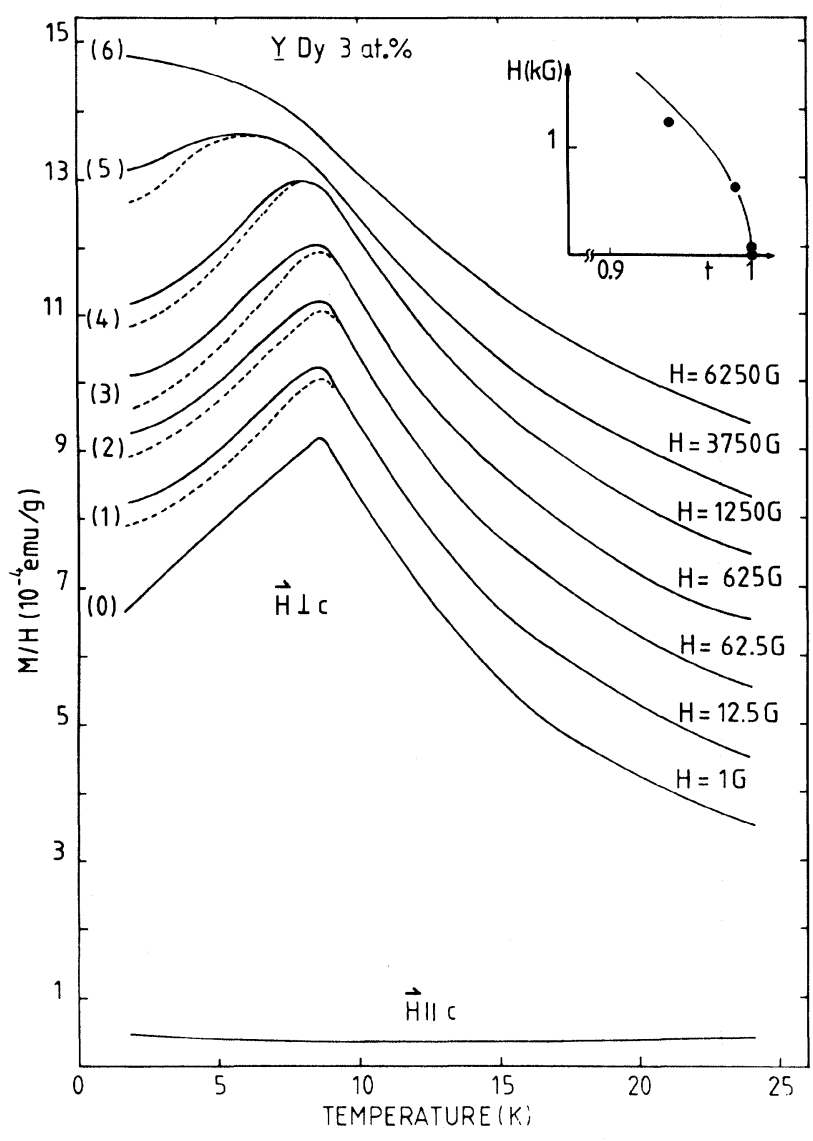

FIG. 5. ZFC (dashed line) and FC (solid line) susceptibilities of $Y$ Dy 3 at. $\%$. Inset: Field $H$ vs $t=\Theta / T_{g}$, where $\Theta$ is defined as the temperature where the ZFC curve departs from the FC one. The full line corresponds to $H^{2} \sim 1-t$.

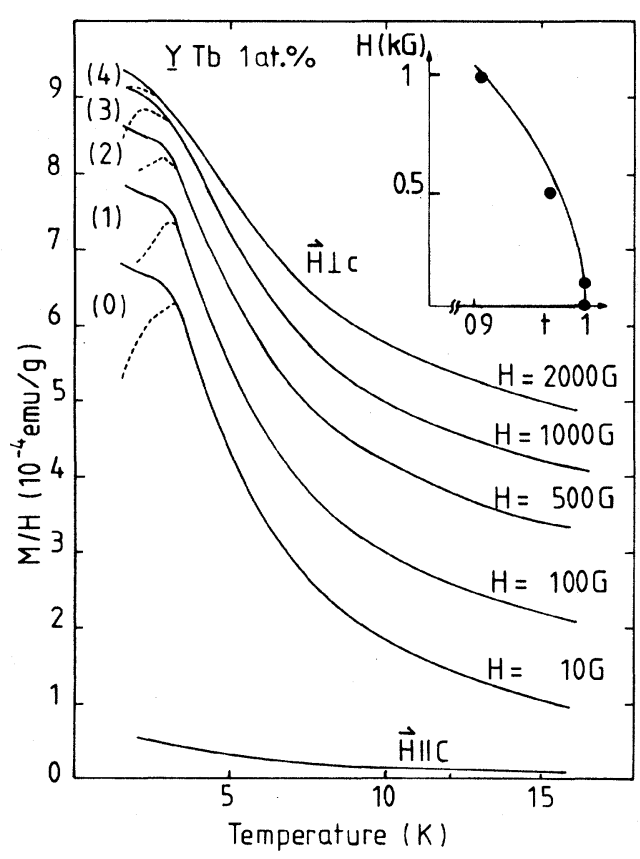

FIG. 6. As Fig. 5 but for $Y$ Tb 1 at. $\%$.
$|D| / j$ is small.

In the Y-based alloys a maximum of the ZFC susceptibility and a splitting of the FC and ZFC curves at low temperature is observed only in transverse fields. In longitudinal field there is no susceptibility maximum, and the FC and ZFC curves coincide down to the lowest experimental temperatures.

In the Sc-based alloys a maximum of the ZFC susceptibility and a splitting between the FC and ZFC susceptibilities is observed in both longitudinal and transverse fields. Again the temperature $(\Theta)$ of the splitting between the FC and ZFC curves is the same (within the experimental error) in longitudinal and transverse fields. The temperature $\left(\Theta^{\prime}\right)$ of the maximum of the ZFC susceptibility is also the same in longitudinal and transverse fields but is different from $\Theta$.

A general feature observed for all our $X$-Y-like alloys is that the temperature $\Theta$ of the splitting between the FC and ZFC susceptibilities is always higher than the temperature $\Theta^{\prime}$ of the maximum of the ZFC susceptibility (Figs. 4-6). This behavior resembles what is predicted for Heisenberg spin-glasses on the basis of the SK Hamiltonian, that is, a splitting between the FC and ZFC curves at the Gabay-Toulouse (GT) temperature, ${ }^{13}$ corresponding to the applied field and a maximum of the ZFC curves near the Almeida-Thouless temperature (see Fig. 7). Moreover, we find (see inset of Figs. 5 and 6) that the field dependence of $\Theta$ corresponds approximately to $1-\Theta / T_{g} \sim H^{2}$, in agreement with what is expected for the GT line. Thus it appears that our $X$ - $Y$-like systems follow the behavior predicted for $m \geq 2$. In contrast, our $S c \mathrm{Er}$ and $Y \mathrm{Er}$ follow the behavior expected for Ising spin-glasses (see Fig. 7), with a splitting between the FC and the ZFC curves, a maximum of the ZFC curve at the same $\Theta$, and $1-\Theta / T_{g} \sim H^{2 / 3}$. However, it is difficult to understand why the behavior predicted for Heisenberg spin-glasses should be observed in $X-Y$ spin-glasses and not in $S c \mathrm{Gd}$ or in the classical Heisenberg spin-glasses such as $\mathrm{CuMn}, \mathrm{AgMn}$, etc.

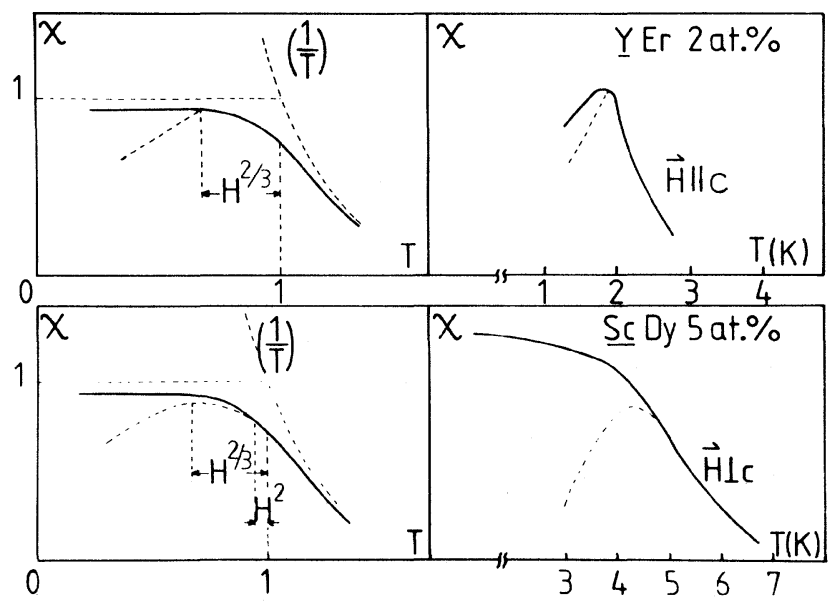

FIG. 7. Schematic difference between the susceptibility of the Ising and Heisenberg infinite-range models around $T_{g}$ [from Sherrington (Ref. 16)]. Experimental examples for Ising-like (Er) and $X-Y$-like (Dy) systems are shown on the right-hand side. 


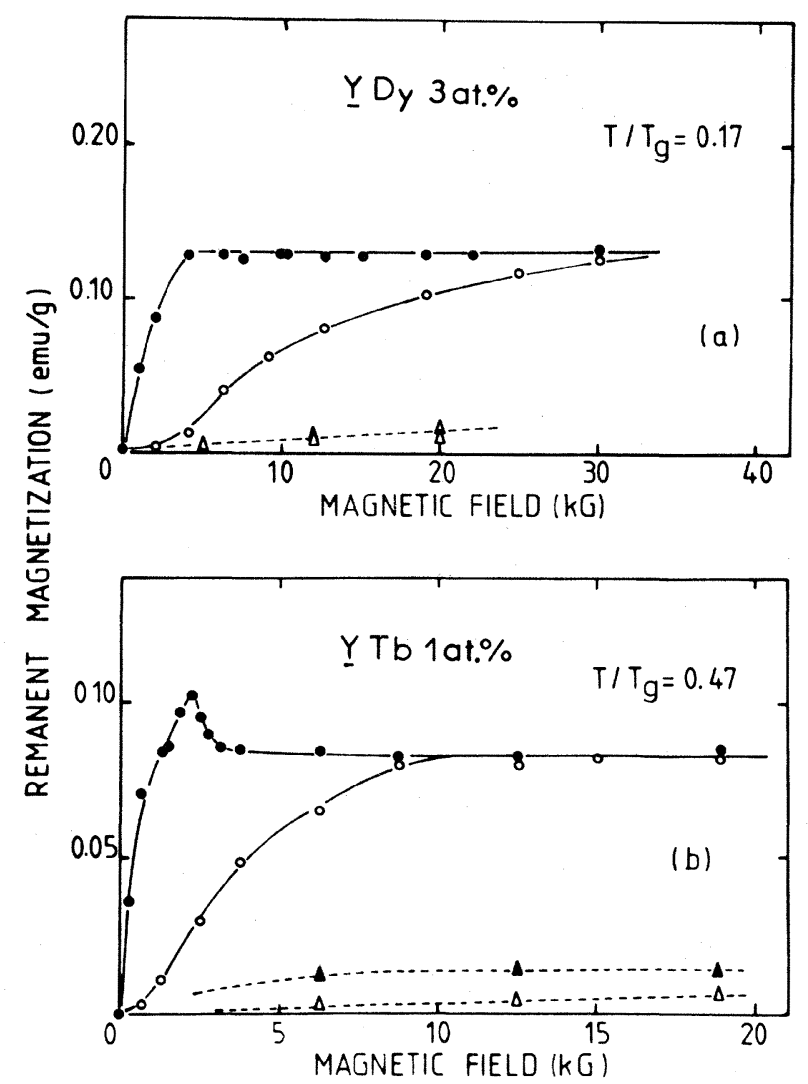

FIG. 8. TRM (closed symbols) and IRM (open symbols) for (a) $Y$ Dy 3 at. $\%$ and (b) $Y$ Tb 1 at. $\%$ in transverse (circles) or longitudinal (triangles) fields.

We have also studied the remanence properties of some $\mathrm{Y}$ - and Sc-based alloys. The results on $Y$ Er have already been presented in Refs. 4 and 6 . We show in Fig. 8 the TRM and IRM of $Y$ Dy and $Y$ Tb alloys for $H$ in the basal plane. The variation of the TRM and the IRM as a function of $H$ is that usually observed in spin-glasses. For $H$ parallel to the $c$ axis the remanences of these two alloys are vanishingly small.

\section{ScGd and YGd (Heisenberg-like alloys)}

The $S c G d$ system exhibits typical spin-glass properties: a sharp cusp of the ZFC susceptibility in low fields, strong broadening of the cusp in moderate fields, and splitting of the FC and ZFC susceptibility curves below the temperature of the cusp. Its susceptibility is nearly, but not completely, isotropic.

The YGd system appears to be a more complex disordered magnetic system because it exhibits a sharp cusp but does not show irreversibility properties below the temperature of the cusp. Its magnetic behavior is also weakly anisotropic.

We plan to report on the magnetic properties of $S c G d$ and YGd and discuss the origin of their weak anisotropy in a future publication. ${ }^{17}$ In this paper we will only compare their freezing temperature with that of the other Scand Y-based alloys (next section).
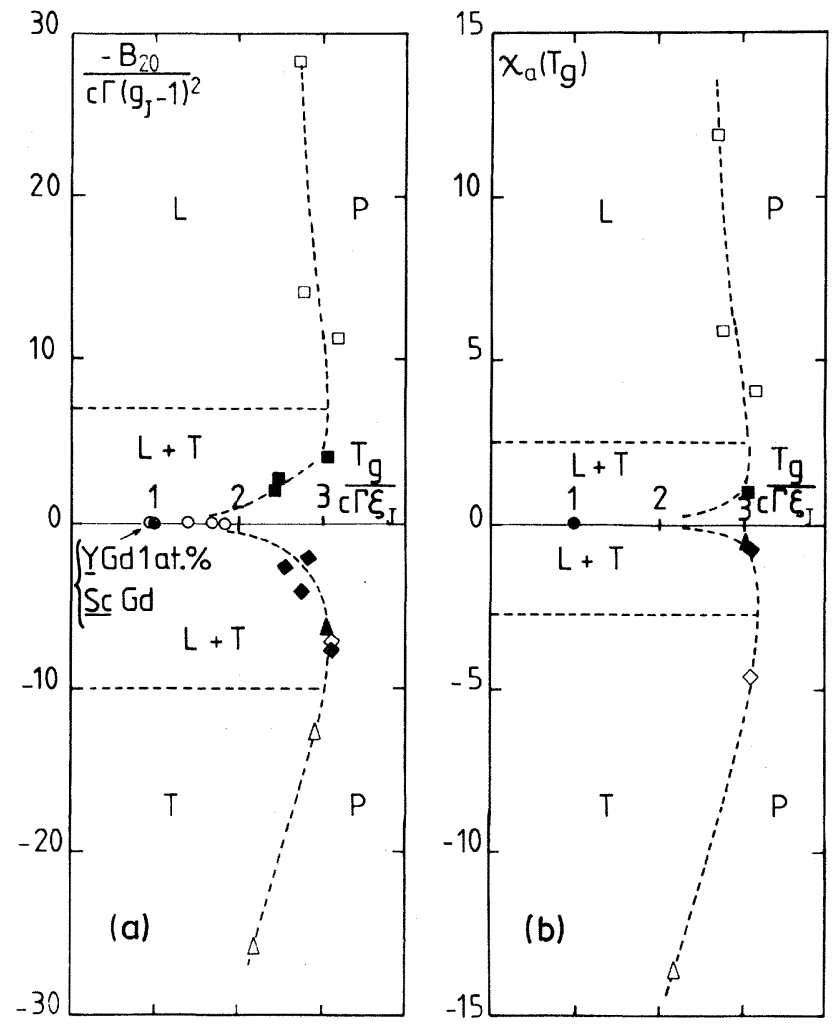

FIG. 9. Experimental phase diagram for the Sc- and Y-based alloys (to be compared to the theoretical phase diagram of Fig. 1). (a): $-B_{2}^{0} /\left[c \Gamma\left(g_{J}-1\right)^{2}\right] \equiv D / j$ vs $T_{g} /\left(c \Gamma \xi_{j}\right) \equiv T_{g} / j$ (see Sec. II for the notation). (b): Experimental anisotropy-exchange ratio [see Eq. (11)] vs $T_{g} /\left(c \Gamma \xi_{J}\right) \equiv T_{g} / j$. The experimental data correspond to $Y \operatorname{Er}(\square), S c E r(\square), Y$ Dy $(\diamond), S c D y(\diamond), Y$ Tb $(\triangle), S c \mathrm{~Tb}(\triangle), Y \mathrm{Gd}(O)$ and $S c \mathrm{Gd}(\bullet)$ alloys. The number of experimental points is smaller in (b) because $\chi_{a}\left(T_{g}\right)$ is known only for single crystals.

\section{Phase diagram}

In Fig. 9(a) we plot the values of $D / j$ vs $T_{g}$ for all our Sc- and Y-based alloys. The values of $D / j$ are calculated as explained in Sec. II. We choose $T_{g}$ as the low-field limit of the temperature at which the FC susceptibility curve departs from the ZFC curve in Figs. 2-6. We have plotted the values of $D / j$ and $T_{g}$ of polycrystalline samples as well as those for the single-crystal alloys studied in the present work. All the values of $D / j$ and $T_{g}$ are listed in Table II. By comparing Fig. 9(a) with the theoretical phase diagram of Fig. 1, one is led to the following conclusions.

(i) The most remarkable agreement of Fig. 9(a) with Fig. 1 is for the ratio of about 3 between the values of $T_{g} / j$ in the Ising and Heisenberg limits, $D / j \gg 1$ and $D / j=0$, respectively. In contrast, the ratio between the experimental values of $T_{g} / j$ in the $X-Y$ and Heisenberg limits is definitely larger than the value 1.5 predicted by theory.

(ii) As $D / j$ varies from large and positive values to large and negative values, the experimental variation of $T_{g}$, with a depression of $T_{g}$ around $D / j=0$, is qualita- 
tively the same as that predicted by theory and represented in Fig. 1. A small difference is the existence of a maximum of $T_{g}$, i.e., the bulge at intermediate values of $|D / j|$.

(iii) At large values of $|D / j|$ [open symbols in Fig. 9(a)] irreversibility effects are observed only for longitudinal (or transverse) fields. At small values of $|D / j|$ [full symbols in Fig. 9(a)] irreversibilities are observed in both directions below the same temperature and our experiments are unable to identify a second transition line at a lower temperature, i.e., the transitions $\mathrm{L} \rightarrow \mathrm{LT}$ or $\mathrm{T} \rightarrow \mathrm{LT}$ in Fig. 1. However, since the theoretical papers Refs. 1 and 2 predicting the second transition do not describe how it can be detected, we cannot completely rule out its existence.

In the above discussion of the properties of the Sc- and Y-based alloys as a function of the parameter $D / j \sim B_{2}^{0} / c$, we have neglected the existence of higher-order crystalfield terms. However, these terms are not completely negligible (see Table I); for example, the term $B_{6}^{6} O_{6}^{6}$ mixes $|J, M\rangle$ and $|J, M \pm 6\rangle$ states and reduces the anisotropy of the spin system. When such effects are significant, the ratio $D / j \sim B_{2}^{0} / c$ is no longer the characteristic parameter of the anisotropy, and it may be better to characterize the anisotropy exchange ratio by the experimental parameter

$$
\chi_{a}\left(T_{g}\right)=\left.\frac{3}{c}\left(\frac{X_{\|}-X_{\perp}}{\chi_{\|}+2 \chi_{\perp}}\right)\right|_{T=T_{g}} .
$$

In Fig. 9(b) we have plotted on the vertical axis the values of $\chi_{a}\left(T_{g}\right)$ instead of those of $D / j$. The graph obtained in this way is not very different from that of Fig. 9(a), and we are led to the same conclusions. Note that
$\chi_{a}\left(T_{g}\right)$ cannot be derived for polycrystalline samples, so only the results on single crystals appear in Fig. 9(b).

\section{E. Mixed alloys: $S c E r D y$ and $Y E r D y$}

In Fig. 10(a) we show the FC and ZFC susceptibility curves of a single crystal of $\mathrm{Y}$ containing 2.2 at. \% of ER and 2 at $\%$ of Dy. The experimental behavior is that of two spin systems which are almost decoupled: The transverse susceptibility presents a cusp at $5 \mathrm{~K}$ and is approximately that of an alloy $Y$ Dy 2 at. \% for which $T_{g}$ is expected to be about $6 \mathrm{~K}$. There is no anomaly at $5 \mathrm{~K}$ in longitudinal fields. The longitudinal susceptibility shows a cusp at about $1.6 \mathrm{~K}$ [see inset of Fig. 10(a)] and is approximately that of an alloy $Y$ Er 2.2 at. \% for which $T_{g}$ is expected to be about $2 \mathrm{~K}$.

Figure 10(b) shows the susceptibility of a polycrystalline sample of Sc containing 12 at. \% Er and 3 at. \% Dy. The freezing temperatures of $S c \mathrm{Er} 12$ at. \% and $S c D y 3$ at. \% would be 3.5 and $2.7 \mathrm{~K}$, respectively. The susceptibility of the ternary alloy exhibits a unique cusp at $T_{g} \simeq 6$ $\mathrm{K}$, which is approximately the sum of 3.5 and $2.7 \mathrm{~K}$. This additivity implies a coupling of the Er and Dy spin systems.

Viana and Bray ${ }^{15}$ have extended their earlier model ${ }^{2}$ to the case of spin-glasses with mixed uniaxial anisotropies. They predict different freezing temperatures for the longitudinal and transverse components in alloys such as ours. The experimental results on $Y$ ErDy are consistent with this prediction and, more generally, with the idea that in the limit of strong anisotropies spins with only longitudinal components and spins with only transverse components cannot be coupled by isotropic exchange. On the other hand, our results on $S c E r D y$ suggest a unique freez-
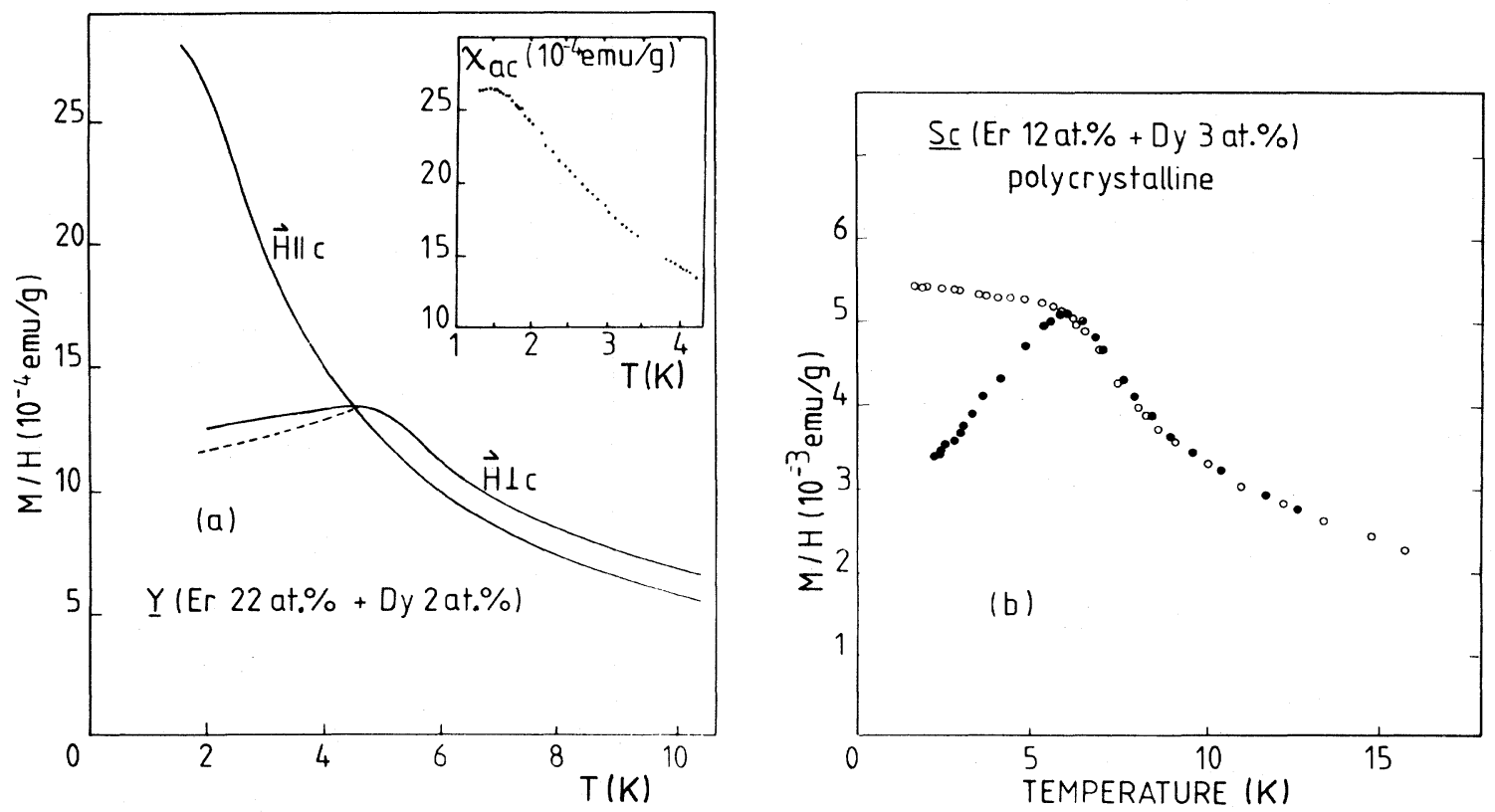

FIG. 10. FC [solid lines in (a) and open circles in (b)] and ZFC [dashed lines in (a) and full circles in (b)] susceptibilities (a) for a single crystal of $\mathrm{Y}$ with 2.2 at. \% of Er and 2 at. \% of Dy and (b) for a polycrystal of Sc with 12 at. \% of Er and 3 at. \% of Dy. Inset: ac susceptibility of the sample of ScErDy in longitudinal fields. 
ing temperature in this alloy; this disagrees with the predictions of Viana and Bray. However, independent measurements of the longitudinal and transverse susceptibilities in a single crystal of $S c E r D y$ would be of interest to confirm that there is a unique freezing temperature.

\section{CONCLUSIONS}

We have investigated the magnetic properties of single crystals of Sc and $\mathrm{Y}$ containing rare-earth impurities and have compared our results with the predictions of the models of Sherrington and Cragg ${ }^{1}$ and Roberts and Bray ${ }^{2}$ for uniaxial spin-glasses.

An interesting result is the resemblance of the theoretical (see Fig. 1) and experimental (see Fig. 9) phase diagrams. There is a remarkable agreement of our results with theory for the ratio 3 between the values of $T_{g} / j$ in the Ising and Heisenberg limits. On the other hand, the agreement with theory is only qualitative when one compares the values of $T_{g} / j$ in $X-Y$ and Heisenberg limits. We point out that the dependence of $T_{g} / j$ on spin dimension seems to appear also in the $Z n \mathrm{Mn}$ (Ising-like, $T_{g} / c \cong 22 \quad \mathrm{~K} /$ at. $\left.\%\right), \quad C d \mathrm{Mn} \quad\left(X-Y\right.$-like,$\quad T_{g} / c \cong 11$ $\mathrm{K} /$ at. $\%$ ), and $M g \mathrm{Mn}$ (Heisenberg-like, $T_{g} / c \cong 3$ $\mathrm{K} /$ at. \%) spin-glasses. ${ }^{3}$ However, a quantitative comparison is questionable for these spin-glasses with different host metals.

Another striking result is the difference between the susceptibility-versus-temperature curves of the Ising-like and $X-Y$-like alloys. In the Ising-like alloys, at the temperature $\Theta$ where the ZFC curve departs from the FC, one finds the maximum of the ZFC susceptibility, and $\Theta$ depends approximately on the applied field according to $H^{2 / 3} \sim 1-\Theta / T_{g}$. This is what is expected for Ising SK spin-glasses. ${ }^{16}$ In contrast, in the $X-Y$-like alloys, $\Theta$ is higher than the temperature of the maximum of the ZFC susceptibility and depends approximately on $H$ according to $H^{2} \sim 1-\Theta / T_{g}$. When $\Theta$ is identified as the Gabay-
Toulouse temperature, this is what is expected for $m \geq 2$ spin-glasses (see Fig. 7 and Ref. 16). If this interpretation is correct, it would be the first experimental identification of the GT line. However, it remains to explain why this behavior is found in our $X-Y$ systems and not in classical Heisenberg spin-glasses such as $\mathrm{CuMn}$.

The last question to answer is the following: Are the Y $\mathscr{R}$ alloys (where $\mathscr{R}$ represents a rare-earth element) good spin-glasses in spite of their tendency to form a helical or modulated magnetic structure? According to Sarkissian and Coles $^{18}$ the $Y \mathscr{R}$ alloys are spin-glasses at low concentrations (in our concentration range) and develop longrange order above 4 or 5 at. \%. However some recent experiments have found characteristic properties of some sort of long-range order in $Y \mathrm{Gd}$, down to 1 at. $\%:^{19} \mathrm{Al}-$ though we cannot completely rule out a trace of longrange order in our $Y \mathrm{Er}, Y \mathrm{Dy}, Y \mathrm{~Tb}$ alloys, our basic assumption in this paper has been to neglect such effects and to consider our Y-based alloys as "uniaxial spinglasses." Note that the Sc-based alloys are much less suspect to develop some long-range order: $S c G d$, in contrast to $Y G d$, exhibits very classical spin-glass properties and, according to Sarkissian and Coles, ${ }^{18}$ helical ordering appears only above approximately 23 at. \% in $\mathrm{ScGd}$ or $\mathrm{ScTb}$.

\section{ACKNOWLEDGMENTS}

We thank H. Bouchiat, I. A. Campbell, M. Gabay, P. Monod, and P. Touborg for many helpful discussions. We thank P. Touborg for providing us with one of the samples. We thank W. Wisny, Freie Universität Berlin (FUB) for performing some of the measurements and C. Kähler (FUB) for growing some of the single crystals. One of us (K.B.) would like to thank the Laboratoire de Physique des Solides for its hospitality during his stay at Orsay. This work was supported in part by Deutsche Forschungsgemeinschaft, Sonderforschungsbereich 161.
"Permament address: Institute für Atom und Festkörperphysik, Freie Universität Berlin, Arnimallee 14, D-1000 Berlin 33, West Germany.

${ }^{1}$ D. M. Cragg and D. Sherrington, Phys. Rev. Lett. 49, 1120 (1982); D. Sherrington, D. M. Cragg, and D. J. Elderfield, J. Magn. Magn. Mater. 31-34, 1417 (1983).

${ }^{2}$ S. A. Roberts and A. J. Bray, J. Phys. C 15, L 527 (1982); A. J. Bray and L. Viana (unpublished).

${ }^{3}$ H. Albrecht, E. F. Wassermann, F. T. Hedgcock, and P. Monod, Phys. Rev. Lett. 4, 819 (1982).

${ }^{4}$ A. Fert, P. Pureur, F. Hippert, K. Baberschke, and F. Bruss, Phys. Rev. B 26, 5300 (1982).

${ }^{5}$ K. D. Usadel, K. Bien, and H. J. Sommers, Phys. Rev. B 27, 6957 (1983).

${ }^{6}$ R. Wendler and K. Baberschke, Solid State Commun. 48, 91 (1983).

${ }^{7}$ D. Sherrington and S. Kirkpatrick, Phys. Rev. Lett. $\underline{35}, 1972$ (1975).

8J.R.L. de Almeida, R. C. Jones, J. M. Kosterlitz, and D. J. Thouless, J. Phys. C 11, L871 (1978).

${ }^{9}$ P. Touborg, Phys. Rev. B 16, 1201 (1977); Ph.D. thesis, Universität Darmstadt, 1978 (unpublished).

${ }^{10}$ G. Keller and J. M. Dixon, J. Phys. F $\underline{6}, 819$ (1976); G. Keller,
Ph.D. thesis, Universität Odense, 1975 (unpublished).

${ }^{11}$ A. Abragam and B. Bleaney, Electron Paramagnetic Resonance of Transition Ions (Oxford University Press, New York, 1970).

${ }^{12} \mathrm{~A}$ careful study of the AT line of $Y \mathrm{Er}$ has been done independently by $\mathrm{H}$. Bouchiat and P. Monod (unpublished).

13J.R.L. de Almeida and D. J. Thouless, J. Phys. A 11, 983 91978); M. Gabay and G. Toulouse, Phys. Rev. Lett. 47, 201 (1981).

${ }^{14}$ There is a principle ambiguity in adapting the classical spin $s$ (from theory) to the quantum-mechanical operator $\overrightarrow{\mathbf{J}}$ or $\overrightarrow{\mathbf{S}}$. Whether one adjusts for equal Zeeman splitting or equal susceptibility the prefactor of $h$ changes.

${ }^{15}$ L. Viana and A. J. Bray (unpublished).

${ }^{16 D}$. Sherrington, in Heidelberg Colloquium on Spin Glasses, Vol. 192 of Lecture Notes in Physics, edited by J. L. van Hemmen and I. Morgenstern, (Springer, New York, 1983, p. 125.

${ }^{17}$ R. Wendler, P. Pureur, A. Fert, and K. Baberschke, J. Magn. Magn. Mater. (in press).

${ }^{18}$ B.V.B. Sarkissian and B. R. Coles, Commun. Phys. 1,17 (1976).

${ }^{19}$ L. E. Wenger and J. A. Mydosh, J. Appl. Phys. (in press). 